\title{
Development of an intracanal mature Enterococcus faecalis biofilm and its susceptibility to some antimicrobial intracanal medications; an in vitro study
}

Shehab El-Din Mohamed Saber

Soha A. El-Hady²

\section{ABSTRACT}

Objectives: To develop a mature biofilm of Enterococcus faecalis inside the root canal system and to test its susceptibility to some antimicrobial medications in vitro.

Methods: Single rooted premolars were mechanically enlarged, sterilized, and then infected with a clinical isolate of E. faecalis. Biofilm formation and maturation was monitored using SEM. Biofilm bacteria were exposed to Amoxicillin+clavulanate, Ciprofloxacin, Clindamycin, Doxycycline, and calcium hydroxide as intracanal medications for 1 week. Finally bacterial samples were collected, and colony-forming units were enumerated.

Results: SEM examination confirmed the formation of a mature biofilm at the end of the incubation period. All the chemotherapeutic agents used were significantly better than Calcium hydroxide in elimination of biofilm bacteria. The antimicrobial effect of Amoxicillin + clavulanate, Ciprofloxacin and Clindamycin was significantly better than Doxycycline $(P=.05)$. However the difference in the antimicrobial effectiveness among them was statistically non-significant $(P=.05)$.

Conclusions: The method used for bacterial biofilm development and maturation is reliable and can be used to assess the anti bacterial potential of endodontic materials. Also, the local application of antibacterial agents can be beneficial in resistant cases of apical periodontitis but only after careful culture and sensitivity testing to choose the appropriate agent for the existing flora. (Eur J Dent 2012;6:43-50)

Key Words: Endodontics; E. faecalis; biofilm

1 Department of Endodontic, Faculty of Dentistry, Ain Shams University, Cairo, Egypt.

${ }_{2}$ Microbiology and Immunology Department, Faculty of Medicine, Ain Shams University, Cairo, Egypt.

Corresponding author: Dr. Shehab El-Din Mohamed Saber, Department of Endodontics. Faculty of Dentistry, Ain Shams University. Cairo. Egypt

Mailing address: El-Rehab city. (81-11-11), 11841,

Cairo, Egypt

Phone: 002-0101413734 Fax: 002-0224835312

E-mail: Shehab007dyahoo.com

\section{INTRODUCTION}

Enterococcus faecalis (E. faecalis) are normally found in the human intestine, but may temporarily be found in the oral cavity, where they have been associated with pathogenic oral manifestations such as mucosal lesions in immunocompromised patients, ${ }^{1}$ as superinfecting organisms in periodontitis ${ }^{2}$ and, most importantly, in persistent root canal infections. ${ }^{3-5}$ 
As facultative organisms, enterococci are exceedingly hardy. They tolerate a wide variety of growth conditions, including temperatures of $10^{\circ} \mathrm{C}$ to $45^{\circ} \mathrm{C}$ and hypotonic, hypertonic, acidic, or alkaline environments. ${ }^{6}$ Several studies have shown that enterococci resist various intracanal treatment procedures. ${ }^{7-8}$ This is attributed for their ability to penetrate dentinal tubules, ${ }^{9}$ withstand high $\mathrm{pH}$ values, ${ }^{10}$ possess virulence factors ${ }^{11}$ and because of biofilm formation. ${ }^{12}$

Biofilms offer their member cells several benefits, the foremost of which is antimicrobial tolerance. Four mechanisms that confer this tolerance to cells living in a biofilm have been suggested. The first is the physical barrier properties of the extracellular polysaccharide matrix..$^{13}$ The second is the physiological state of biofilm microorganisms. Bacterial cells residing within a biofilm grow more slowly than planktonic cells; as a result, biofilm cells take up antimicrobial agents more slowly. ${ }^{14}$ Furthermore, the depletion of nutrients can forces bacteria into a dormant or stationary growth phase in which they are protected from being killed. ${ }^{15}$ The third suggested mechanism is metabolic heterogeneity. Cells located more deeply in the biofilm are exposed to environmental conditions that differ from those at the surface including decreased oxygen tension. This results in altered phenotypes in terms of growth rates and gene transcription that might facilitate certain survival and virulence characteristics. ${ }^{16}$ Finally, it has been speculated that a sub-population of microorganisms known as persisters exists. These microorganisms constitute a small percentage of the original population and are believed to constitute a highly resistant phenotypic state that is resistant to being killed by antimicrobial agents. ${ }^{17}$

Management of persistent root canal infections is a challenging task complicated by the complex anatomy of the root canal system. Several antibacterial strategies have been described to reduce the number of microorganisms in infected root canal systems, including the use of various instrumentation techniques, irrigation regimens, intracanal medicaments and, sometimes, systemic antibiotics.

Although systemic antibiotics appear to be an effective adjunct in certain surgical and nonsurgical endodontic procedures, their administration is not without the potential risk of adverse systemic effects, such as allergic reactions, toxicity and the development of resistant strains of microbes.

In addition, the systemic administration of antibiotics relies on patient compliance with the dosing regimens, followed by absorption through the gastro-intestinal tract and distribution via the circulatory system to bring the drug to the infected site. ${ }^{18}$ Unfortunately, the necrotic root canal is a secluded cavity inaccessible to the local immune system, ${ }^{19}$ and the concentration of drug that reaches the canal space after systemic administration of antibiotics is minimal and unlikely to inhibit bacterial growth. ${ }^{20}$ Therefore, local application of antibiotics within the root canal system may be a more effective mode for delivering the drug. ${ }^{21-23}$

Selection of the appropriate local antibiotic to be used in persistent endodontic infections is also challenging because the prevailing species, $E$. faecalis, has demonstrated resistance to multiple antimicrobial agents. ${ }^{24}$ This resistance is either intrinsic or extrinsic. The intrinsic resistance is chromosomally mediated and present in all members of the species; examples involve resistance to penicillin G, ampicillin, cephalosporin, aminoglycosides, trimethoprim, and sulfamethazole. On the other hand, the acquired resistance results from either DNA mutation or acquisition of new DNA; examples involve resistance to almost all other antimicrobial agents such as B-lactams, aminoglycosides, fluoroquinolones, macrolides, azalides, tetracycline and vancomycin. ${ }^{25}$

Recent laboratory studies have attempted to evaluate the efficacy of antimicrobial agents used in root canal treatment against $E$. faecalis grown as a biofilm ${ }^{26}$ rather than relying on planktonic culture models because planktonic bacteria do not generally represent the in vivo growth condition found in infected root canal systems. However, the time taken for biofilm formation varied considerably among this studies. ${ }^{27}$ Theoretically, an older biofilm will be more mature and more difficult to eliminate. Therefore, the purpose of this study was to assess the susceptibility of mature E. faecalis biofilms to some locally applied antimicrobial agents in vitro that can be a useful addition to the antibacterial strategies used in the treatment of persistent root canal infections.

\section{MATERIALS AND METHODS}

Selection and Preparation of the Samples

One hundred forty-eight human mandibular premolars with mature apices and single root canals were selected for use in this study. All teeth were stored in $0.5 \%$ thymol solution at $4^{\circ} \mathrm{C}$ before use. Following periapical radiographs, the crowns 
were removed using a safe sided diamond disc (NTI diamond disc, Axis Dental, USA) mounted on a high speed contra-angle with water coolant, and the tooth length was standardized to $18 \mathrm{~mm}$ from the root apex to the coronal border. Cleaning and shaping of samples was performed using the Protaper rotary nickel titanium system (Dentsply Maillefer, Ballaigues, Switzerland) in the presence of $2.5 \%$ $\mathrm{NaOCl}$ until finishing file \# 4 (40/ 0.6). Following preparation, all canals received a final irrigation sequence of $5 \mathrm{ml}$ of $17 \%$ EDTA, followed by $5 \mathrm{ml}$ of $2.5 \% \mathrm{NaOCl}$ and $5 \mathrm{ml}$ of sterile water (Baxter sterile water, Dealmed Medical supplies, USA) to remove the smear layer. The teeth were then air-dried and steam autoclaved at $121^{\circ} \mathrm{C}$ for 30 minutes. The entire root surface, including the apical foramen of each sample, was coated with two layers of nail polish (Max Factor, Cosmetics and Fragrances, London, UK).

\section{Classification of the Samples}

One hundred forty-eight samples were included in the study. One hundred forty samples were divided into five experimental groups and two control groups ( $n=20)$. The five experimental groups represented the antimicrobial agents used;

Group 1: Amoxicillin and clavulanate (Augmentin, Medical Union Pharmaceuticals, Egypt for GSK).

Group 2: Ciprofloxacin (Ciprobay ${ }^{\circledR 500}$ Bayer Healthcare, AG. Germany).

Group 3: Clindamycin (Clindam 150 Sigma Pharmaceutical industries. Egypt)

Group 4: Doxycycline (Doxymycin ${ }^{100}$ Nile company for Pharmaceuticals and Chemical Industries. Egypt)

Group 5: Calcium hydroxide paste (Control ${ }^{\circledR}$ La Maison Dentaire S.A.Balzers)

The positive control group was used to check for bacterial viability throughout the experiment, while the negative control group was used to check for sterility of the procedures. The remaining eight samples were used as indicators for biofilm formation.

\section{Biofilm Development}

A clinical isolate of E. faecalis from the Microbiology Laboratory (Central Laboratories, Ministry of Health, Egypt) was used for biofilm formation. Samples from the experimental groups and the positive control group were immersed in a 24hour pure culture suspension of E. faecalis grown in Brain Heart Infusion broth (BHI; Difco Labo- ratories, Detroit, MI, USA) and adjusted to No. 1 MacFarland turbidity standard; all teeth were incubated at $37^{\circ} \mathrm{C}$ in sealed vials. This procedure was repeated every 72 hours using a 24-hour pure culture prepared and adjusted to the No. 1 MacFarland turbidity standard. The negative control samples were immersed in sterile $\mathrm{BHI}$ broth replenished with sterile saline every 72 hours to test for sterility of the procedures. The teeth were maintained in a humid environment at $37^{\circ} \mathrm{C}$ for 30 days.

\section{Verification of Biofilm Development}

Monitoring of bacterial biofilm development onto root canal dentin was assessed by SEM examination at four time intervals $(3,10,20$ and 30 days). Longitudinal grooves were cut along the entire length of eight randomly selected samples before root canal preparation. Then, after immersion in the bacterial suspension, two samples were split with a hammer and chisel into two halves as described by Sen et a ${ }^{28}$ at each time interval. Each half was immersed in 2.5\% glutaraldehyde (pH 7.4) for 24 hours at $4^{\circ} \mathrm{C}$ for fixation, washed with phosphate buffer saline for $15 \mathrm{~min}$, and postfixed in $1 \%$ (wt/voll) osmium tetroxide for 12 hours at $4^{\circ} \mathrm{C}$. Then, the samples were flushed with PBS, and then dehydrated in an ascending acetone series $130 \%, 60 \%$, and $100 \%$ ) for 10 minutes each. Finally, the samples were dried by using a SAMDRI PVT-3 critical point dryer apparatus (Tousimis Research Corp., Rockville, MDl using liquid $\mathrm{CO}_{2}$ replacement. Each sample was mounted and sputter coated (208HR High Resolution Sputter Coater. Ted Pella Inc,USA) with a $200 \AA$ layer of gold palladium. Observation of the whole canal was performed by using a JEOL JSM-35CF scanning electron microscope at $30 \mathrm{kV}$.

Preparation and Application of the Antimicrobial Agents

The drug concentrations were adjusted based on published data for minimum inhibitory concentrations for E. faecalis. ${ }^{29}$ Sterile saline solution (Al Mottahedoon Pharma, 10th Ramadan City, CairoEgypt) was added in a drop-wise manner to the antibiotic powder and mixed to obtain a thick paste. The MTA carrier (Dentsply) was used to introduce the paste inside the infected canals. To allow the antibacterial properties of the intracanal medicaments to be expressed under clinical conditions, wax was used to seal the apex as well as the coronal access cavity; then aluminium foil was used to 
envelop the samples. All samples were incubated for a week at $37^{\circ} \mathrm{C}$ under humid conditions.

\section{Bacterial Sampling}

After one week, all of the samples were irrigated with $20 \mathrm{ml}$ sterile saline solution to remove the root canal contents. Bacterial samples were taken using a standard method of collection. ${ }^{30}$ The root canals were filled with sterile saline as a transport fluid, then \#15 K-file was placed into the canal to within $1 \mathrm{~mm}$ of working length and circumferentially filed for 10 seconds before sterile absorbent paper points adsorbed the transport fluid and transferred it to a test tube containing $1.0 \mathrm{ml}$ of saline. All samples were vortexed for twenty seconds and 10 -fold dilutions were prepared in saline. Aliquots of $0.1 \mathrm{ml}$ were spread plated onto $\mathrm{BHI}$ agar plates, incubated at $37^{\circ} \mathrm{C}$ for 48 hours, and colony-forming units (CFU) per $1 \mathrm{~mL}$ were enumerated.

\section{Statistical analysis}

Statistical analysis was carried out using SAS program (SAS, Statistical Analysis Systems, STAT/ User's Guide, Release 6.03 ed., SAS Institute, Cary NC, USA.1988). Colony forming units were transferred using log transformation before statistical analysis. One Way Analysis of Variance (Procedure ANOVA of SAS) followed by Duncan's Multiple Range Test were used to test the effect of treatments on colony forming units.

\section{RESULTS}

SEM examination at the specified time intervals (Figure 1a-d) showed that E. Faecalis consistently adhered to collagen structures, colonized dentin surfaces, progressed toward the dentinal tubules, and formed a mature biofilm at the end of the incubation period.

For the negative control group, these previously non-infected samples showed no growth. In contrast, the positive controls showed positive growth in all cultured samples. For the experimental groups (Table 1), there was a statistically significant reduction in the mean numbers of colony-forming units $(P=.05)$ after a 1 -week application of the antimicrobial agents. However, none of them resulted in complete elimination of biofilm bacteria. All of the chemotherapeutic agents used were significantly better than Calcium hydroxide $10 \%$ negative cultures). The antimicrobial effect of Amoxicillin + clavulanate $(80 \%$ negative cultures), Ciprofloxacin $(80 \%$ negative cultures) and Clindamycin $(50 \%$ negative cultures) was significantly better than for Doxycycline (30\% negative cultures) at $P=.05$. How- ever, the difference in the antimicrobial effectiveness among them was statistically non-significant $(P=.05)$.

\section{DISCUSSION}

The experimental model used in this study is concerned to mimic some clinical cases in which the clinicians have already completed the mechanical preparation, used all types of available irrigant combinations and the patients took more than one class of systemic antibiotics, but still there are some persistent clinical signs and symptoms in the form of slight pain, tenderness to percussion and / or mild exudate.

This clinical picture suggests the presence of a resistant type of extra and / or intra radicular infection. This is in agreement with Chaves de Pas et al ${ }^{31}$ who found that once established, there is a pattern for non-mutans streptococci, enterococci and lactobacilli to survive after chemo-mechanical preparation in root canals with clinical and radiographical signs of apical periodontitis.

Such cases might benefit from the local application of an antibacterial agent. However, this agent should be selected after careful culture and sensitivity testing to identify the existing flora. Thus our model was to develop a mature bacterial biofilm of a recognized endodontic pathogen onto root canal dentin, then to test its susceptibility to some popular antibacterial agents.

The first objective of our study was to develop a bacterial biofilm onto root canal dentin. Introducing the biofilm concept to endodontic microbiology is a major step forward in our understanding of persistent root canal infections. Microorganisms growing in biofilms are better protected from adverse environmental changes and antimicrobial agents, in some cases up to 1,000-fold greater than that of the same microorganisms living planktonically. ${ }^{32}$

Recent studies used several protocols to evaluate the efficacy of selected irrigants and medicaments to remove biofilms grown in wells, ${ }^{33}$ on membrane filters ${ }^{34}$ and on dentin samples. ${ }^{35-37}$ Esterala et $\mathrm{al}^{27}$ suggested that three aspects must be considered for validation of biofilm models: the bacterial colonization structure, the biological indicator, and the time necessary for biofilm formation.

All of these aspects were achieved in our study. The bacterial colonization structure was the human root canal with its unique microenvironment. The biological marker was E. faecalis which is a dominant endodontic pathogen with several virulence factors. Finally the infection period used was 
sufficient for development and maturation of the bacterial biofilm as evident by SEM examination. Thus our model can be of benefit to researchers investigating the anti microbial potential of endodontic materials.

Selection of the antimicrobial agents was based on previous studies that tested the susceptibility / resistance patterns of E. faecalis. Penicillin and ampicillin were not selected because exclusive strains of E. faecalis express the B-lactamase en- zyme; ${ }^{38}$ instead, amoxicillin la selective inhibitor of bacterial cell wall synthesis) with clavulanic acid (a B-lactamase inhibitor) was used. Other selected agents were Ciprofloxacin la broad spectrum fluoroquinolone which inhibits microbial nucleic acid synthesis), which is incorporated in a recently popular protocol for disinfection of immature necrotic teeth, ${ }^{39}$ Clindamycin la bacteriostatic lincosamide that inhibits microbial protein synthesis), which is a popular drug used in the treatment of acute peri-

Table 1. Mean colony forming units and the percentages of negative cultures after 1-week application of the antimicrobial agent.

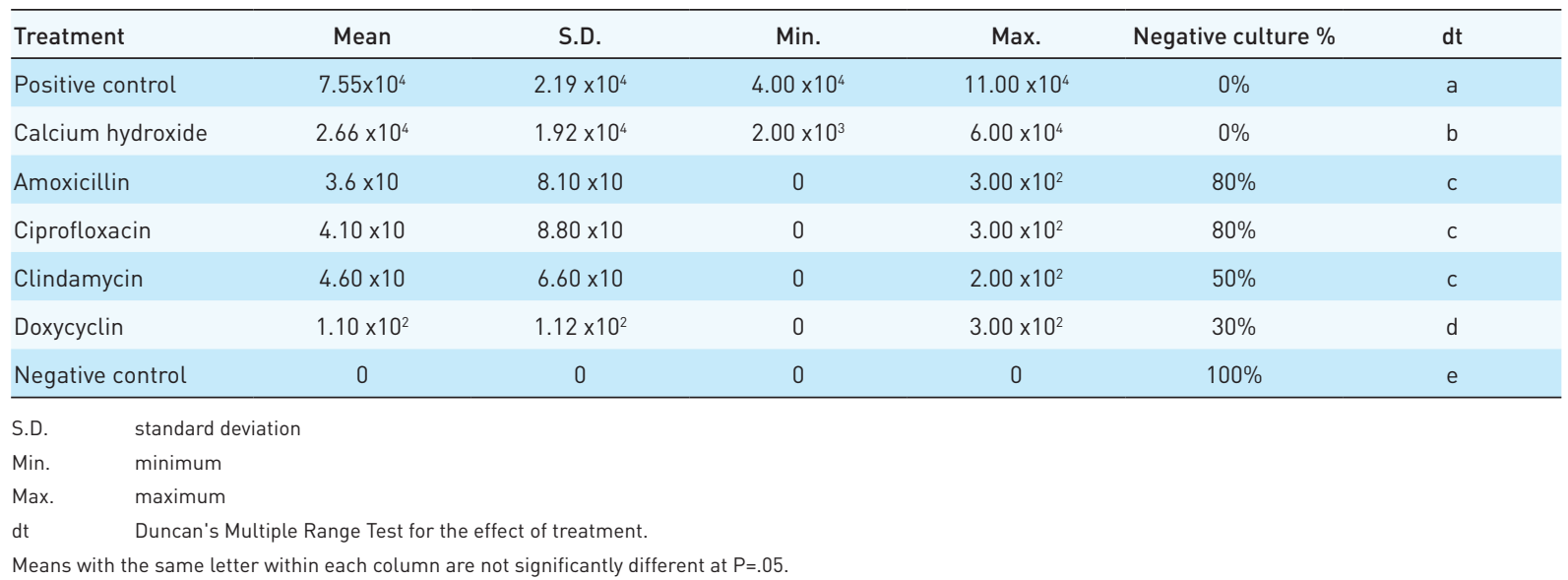
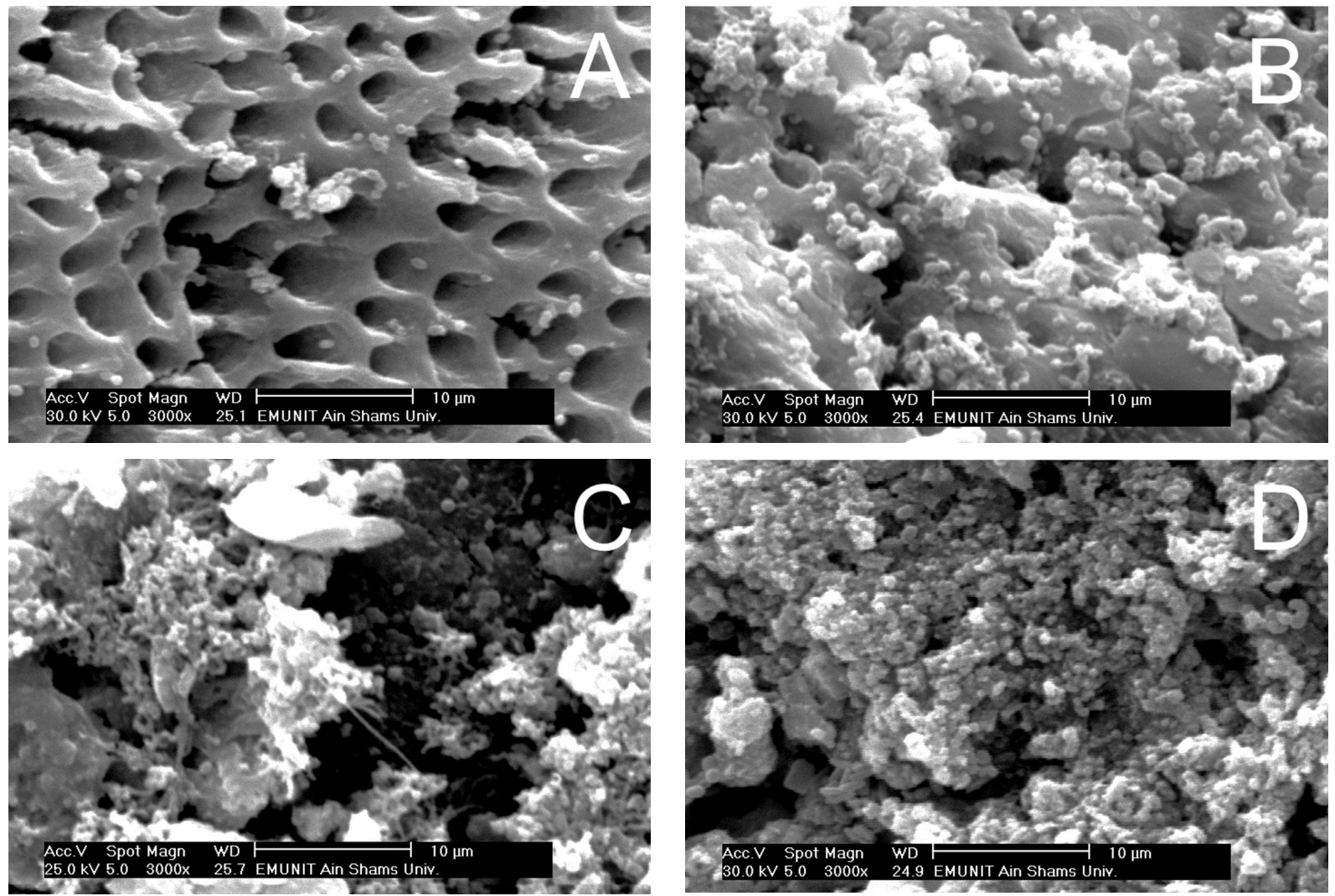

Figure 1 A-D. RSEM images showing the stages of E.faecalis biofilm formation and maturation onto root canal dentin

Figure 1 A. 3-days-old biofilm showing E. faecalis colonizing the dentin surface and starting to invade the patent dentinal tubules.

Figure $1 \mathrm{~B} .10$-days-old biofilm showing more micro colonies of $\mathrm{E}$. faecalis penetrating inside dentinal tubules.

Figure 1 C, D. 30-days-old biofilm showing an increase in the number and density of the bacterial micro colonies to a remarkable thickness that is almost covering the entire dentinal surface. 
apical infections, and in cases of penicillin allergy and Doxycyclin la broad spectrum tetracycline that inhibits, but does not kill susceptible bacterial incorporated in MTAD ${ }^{40}$ and reported to be effective against facultative and anaerobic odontogenic infectious flora. ${ }^{41}$

Calcium hydroxide was also selected because it is the most widely used intracanal medicament. ${ }^{42}$ Its antibacterial effect is attributed to the release of hydroxyl ions. Several studies have demonstrated that after placement of calcium hydroxide in the root canal system, the hydroxyl ions diffuse through the dentinal tubules to the outer surface of the root. ${ }^{43-44}$ On the basis of these reports, it is possible that calcium hydroxide exerts an immunomodulatory effect by local denaturation of inflammatory mediators, possibly via alkaline hydrolysis of amide bonds ${ }^{45}$ or denaturation of some proinflammatory mediators. ${ }^{46}$

The $\mathrm{pH}$ of the formulation selected in our study was 12 because Evans et a ${ }^{47}$ have reported that $E$. faecalis is resistant to killing by calcium hydroxide at or below a $\mathrm{pH}$ value of 11.1 .

The use of instruments for removal of canal contents was avoided so as not to disrupt the biofilms. Passive irrigation only was employed and it proved to be sufficient as evident visually on the paper points used during culturing that had no traces of the agents used. This was probably facilitated by the relatively short length of the roots, the adequate coronal taper of the canals as well as the large volume of saline solution used. This volume most likely did not affect the bacterial biofilms as evident by the high count obtained from the positive control group which was statistically significant in comparison with the other groups.

The presence of E.faecalis in root canals can be detected either by culture or by molecular techniques. Cogulo et al ${ }^{48}$ confirmed that both culture and PCR methods were sensitive to detect E. faecalis in both deciduous and permanent teeth. The culture method was used in this study, it is considered to be useful as a primary investigation method to identify predominant species or make a correlation of some bacteria to certain clinical findings, while molecular techniques can detect uncultivable or difficult-to-grow bacteria or examine more specific effects. However, all results obtained from invitro tests should be interpreted with caution, as they might not demonstrate the full clinical potential of the agents being tested.

Results of this study indicate that all of the chemotherapeutic agents used were significantly better than calcium hydroxide in the elimination of biofilm bacteria. This might be due to the paste form used for calcium hydroxide in this study. Staehle et al $^{49}$ demonstrated that aqueous calcium hydroxide suspension had a higher degree of hydroxyl ion release than the cement or the pastetype calcium hydroxide products. Our results are in agreement with previous studies ${ }^{50-53}$ who reported less bacterial activity of calcium hydroxide against E. Faecalis. However, these findings are in disagreement with Baik et al ${ }^{54}$ who suggested that calcium hydroxide could detoxify lipoteichoic acid, a major virulence factor of gram positive bacteria, resulting in attenuation of the inflammatory response to E.faecalis. This difference may be due to the application of calcium hydroxide to planktonic bacteria in their study rather than to a biofilm structure as in our study where the bacteria can resist alkaline stress. ${ }^{55}$

On the other hand, none of them was able to achieve complete sterilization of the infected root canal system. The standard deviation was relatively high in some groups due to the presence of completely negative cultures in these groups. The higher percentage of negative culture obtained with Amoxicillin+clavulanate and Ciprofloxacin (80\%) over Clindamycin (50\%) and Doxycyclin (30\%) may be because they are bactericidal agents.

In order to obtain the maximum clinical benefit of the anti-bacterial agents used in this mode, additional research should be carried out to investigate the best drug delivery form, drug substantivity and the feasibility of using drug combinations. This would be more practical if the biofilm model was a polymicrobial one. Also possible side effects in the form of sensitization, development of resistant strains or any alteration of root canal dentin surface characteristics should be investigated.

\section{CONCLUSIONS}

The method used for bacterial biofilm development and maturation is reliable and can be used to assess the anti bacterial potential of endodontic materials. Also, the local application of antibacterial agents can be beneficial in resistant cases of apical periodontitis but only after careful culture and sensitivity testing to choose the appropriate agent for the existing flora. 


\section{REFERENCES}

1. Wahlin YB, Holm AK. Changes in the oral microflora in patients with acute leukemia and related disorders during the period of induction therapy. Oral Surg Oral Med Oral Pathol Oral Radiol Endod 1988;65:411-417.

2. Rams TE, Feik D, Young V, Hammond BF, Slots J. Enterococci in human periodontitis. Oral Microbiol Immunol 1992; 7:249-252.

3. Hancock HH 3rd, Sigurdsson A, Trope M, Moiseiwitsch J. Bacteria isolated after unsuccessful endodontic treatment in a North American population. Oral Surg Oral Med Oral Pathol Oral Radiol Endod 2001;91:579-586.

4. Pinheiro ET, Gomes BP, Ferraz CC, Teixeira FB, Zaia AA, Souza Filho FJ. Evaluation of root canal microorganisms isolated form teeth with endodontic failure and their antimicrobial susceptibility. Oral Microbiol Immunol 2003;18:100-103.

5. Siqueira JF, Rôças IN. Polymerase chain reaction-based analysis of microorganisms associated with failed endodontic treatment. Oral Surg Oral Med Oral Pathol Oral Radiol Endod 2004;97:85-94.

6. Huycke MM, Sahm DF, Gilmore MS. Multiple-drug resistant enterococci: the nature of the problem and an agenda for the future. Emerg Infect Dis 1998;4:239-249.

7. Hancock HH, Jr, Sigurdsson A, Trope M, Moiseiwitsch J. Bacteria isolated after unsuccessful endodontic treatment in a North American population. Oral Surg Oral Med Oral Pathol Oral Radiol Endod 2001;91:579-586.

8. Sundqvist G, Figdor D, Persson S, Sjögren U. Microbiologic analysis of teeth with failed endodontic treatment and the outcome of conservative re-treatment. Oral Surg Oral Med Oral Pathol Oral Radiol Endod 1998;85:86-93.

9. Sedgley CM, Lennan SL, Appelbe OK. Survival of Enterococcus faecalis in root canals ex vivo. Int Endod J 2005;38:735742.

10. Portenier I, Waltimo TMT, Haapasalo M. Enterococcus faecalis - the root canal survivor and 'star' in post-treatment disease. Endod Topics 2003;6:135-159.

11. Sedgley CM, Molander A, Flannagan SE. Virulence, phenotype and genotype characteristics of endodontic Enterococcus spp. Oral Microbiol Immunol 2005;20:10-19.

12. Svensater G, Bergenholtz G. Biofilms in endodontic infections. Endod Topics 2004;9:27-36

13. Jett BD, Huycke MM, Gilmore MS. Virulence of enterococci. Clin Microbiol Rev 1994; 7:462-478.

14. Elsner HA, Sobottka I, Mack D. Virulence factors of Enterococcus faecalis and Enterococcus faecium blood culture isolates. Eur J Clin Microbiol Infect Dis 2000;19:39-42.

15. Haapasalo M, Orstavik D. In Vitro infection and disinfection of dentinal tubules. J Dent Res 1987;31:1-7
16. Ten Cate JM. Biofilms, a new approach to the microbiology of dental plaque. Odontology 2006;94:1-9.

17. Chávez de Paz L. Redefining the Persistent Infection in Root Canals: Possible Role of Biofilm Communities. J Endod 2007;33:652-662.

18. Mohammadi Z, Abbott PV. On the local applications of antibiotics and antibiotic-based agents in endodontics and dental traumatology. Int Endod J 2009;42:555-567.

19. Stashenko P, Teles R, D'Souza R. Periapical inflammatory responses and their modulation. Crit Rev Oral Biol Med 1998;9:498-521.

20. Abbott PV, Hume WR, Pearman JW. Antibiotics and endodontics. Aust Dent J 1990;35:50-60.

21. Bystrom A, Claesson R, Sundqvist G. The antibacterial effect of campho-rated paramonochlorphenol, camphorated phenol and calcium hydroxide in the treatment of infected root canals. Endod Dent Traumatol 1985;1:170-175.

22. Gilad JZ, Teles R, Goodson M, White RR, Stashenko P. Development of a clindamycin-impregnated fibre as an intracanal medication in endodontic therapy. $J$ Endod $1999 ; 25: 722-727$.

23. Portenier I, Haapasalo H, Rye A, Waltimo T, Ørstavik D, Haapasalo $M$ Inactivation of root canal medicaments by dentine, hydroxylapatite and bovine serum albumin. Int En$\operatorname{dod} J$ 2001;34:184-188.

24. Martohi YA, Agnihorti H, Dubey D. Enteroccocal resistancean overview. Indian J Med Microbiol 2005;23:214-219.

25. Greenwood D, Slack R, Peutherer J. Antimicrobial agents. In: Medical Microbiology a guide to microbial infection; pathogenesis, immunity, Lab. Diagnosis and control. London: Churchill Livingstone; 2007 pp.52-67.

26. Abdullah M, Ng YL, Gulabivala K, Moles DR, Spratt DA. Susceptibilities of two Enterococcus faecalis phenotypes to root canal medications. J Endod 2005;31:30-36.

27. Esterla C, Sydney GB, Figueiredo JP, Esterla ER. A model system to study antimicrobial stratigies in endodontic biofilms. J Appl Oral Sci 2009;17:87-91.

28. Sen BH, Piskin B, Demirci T. Observation of bacteria and fungi in infected root canals and dentinal tubules by SEM. Endod Dent Traumatol 1995;11:6-9.

29. Andrews JM. Determination of minimum inhibitory concentrations. J Antimicrob Chemother 2001;48:5-16.

30. Edgar S, Marshall JG, Baumgatner JC. The Antimicrobial Effect of Chloroform on Enterococcus faecalis After GuttaPercha Removal. J Endod 2006; 32:1185-1187.

31- Chavez de Paz LE, Dahlen G, Molander A, Moller ,Bergenholtz G. Bacteria recovered from teeth with apical periodontitis after antimicrobial endodontic treatment. Int Endod $J$ 2003;36:500-508. 
32. Johnson SA, Goddard PA, Iliffe C, Timmins B, Rickard AH, Robson G, Handley PS. Comparative susceptibility of resident and transient hand bacteria to para-chloro-meta-xylenol and triclosan. J Appl Microbiol 2002;93:336-344.

33. Dunavant TR, Regan JD, Glickman GN, Solomon ES, Honeyman AL. Comparative evaluation of endodontic irrigants against Enterococcus faecalis biofilms. J Endod 2006; 32:527-531

34. Spratt DA, Pratten J, Wilson M, Gulabivala K. An in vitro evaluation of the antimicrobial efficacy of irrigants on biofilms of root canal isolates. Int Endod J 2001;34:300-307.

35. George S, Kishen A, Song KP. The role of environmental changes on monospecies biofilm formation on root canal wall by Enterococcus faecalis. J Endod 2005;31:867-872.

36. Kishen A, George S, Kumar R. Enterococcus faecalis-mediated biomineralized biofilm formation on root canal dentine in vitro. J Biomed Mater Res A 2006;77:406-415.

37. Duggan JM, Sedgley CM. Biofilm Formation of Oral and Endodontic Enterococcus faecalis. J Endod 2007;33:815818.

38. Murray BE. Beta-Lactamase-producing enterococci. Antimicrob Agents Chemother 1992;36:2355-2359.

39. Windley W 3rd, Teixeira F, Levin L, Sigurdsson A, Trope M. Disinfection of Immature Teeth with a Triple Antibiotic Paste. J Endod 2005;31:439-443.

40. Torabinejad M, Shabahang S, Aprecio R, Kettering JD. The antimicrobial effect of MTAD: an in vitro investigation. $J$ En$\operatorname{dod} 2003 ; 29: 400-403$.

41. Chan $\mathrm{Y}$, Chan $\mathrm{CH}$. Antibiotic resistance of pathogenic bacteria from odontogenic infections in Taiwan. J Microbiol Immunol Infect 2003;36:105-110.

42. Sjögren U, Figdor D, Spångberg L, Sundqvist G. The antimicrobial effect of calcium hydroxide as a short-term intracanal dressing. Int Endod $J$ 1991;24:119-125.

43. Esberard RM, Carnes DL Jr, del Rio CE. Changes in $\mathrm{pH}$ at the dentin surface in roots obturated with calcium hydroxide pastes. J Endod 1996;22:402-405.

44. Nerwich A, Figdor D, Messer HH. pH changes in root dentin over a 4-week period following root canal dressing with calcium hydroxide. J Endod 1993;19:302-306.

45. Voel D, Voel JG, Pratt CW. Fundamentals of biochemistry. 1st ed. Hoboken, NJ: John Wiley \& Sons, 1999:93-123.

46. Khan AA, Sun X, Hargreaves KM. Effect of Calcium Hydroxide on Proinflammatory Cytokines and Neuropeptides. $J$ Endod 2008;34:1360-1363.

47. Evans M, Davies JK, Sundqvist G, Figdor D. Mechanisms involved in the resistance of Enterococcus faecalis to calcium hydroxide. Int Endod J 2002;35:221-228.
48- Cogulu D, Uzel A, Oncag O, Aksoy S, Eronat C. Detection of Enterococcus faecalis in necrotic teeth root canals by culture and polymerase chain reaction Methods. Eur J Dent 2007;1:216-221.

49- Staehle HJ, Pioch T, Hoppe W. The alkalizing properties of calcium hydroxide compounds. Endod Dent Traumatol 1989;5:147-145.

50. Siren EK, Haapasalo MP, Ranta K, Salmi P, Kerosuo EN Microbiological findings and clinical treatment procedures in endodontic cases selected for microbiological investigation. Int Endod J 1997;30:91-95.

51. George S, Kishen A, Song KP. The role of environmental changes on monospecies biofilm formation on root canal wall by Enterococcus faecalis. J Endod 2005;31:867-872.

52- Orstavik D, Haapasalo M. Disinfection by endodontic irrigants and dressings of experimentally infected dentinal tubules. Endod Dent Traumatol 1990;6:142-149.

53- Heling I, Steinberg D, Kenig S, et al. Efficacy of a sustainedrelease device containing chlorhexidine and $\mathrm{Ca}(\mathrm{OH}) 2$ in preventing secondary infection of dentinal tubules. Int Endod $J$ 1992;25:20-24.

54- Baik JE, Kum KY, Yun CH, Lee JK, Lee K, Kim KK, Han SH. Calcium hydroxide inactivates lipoteichoic acid from Enterococcus faecalis. J Endod 2008;34:1355-1359.

55- Brandle N, Zehnder M, Weiger R, Waltimo T. Impact of growth conditions on susceptibility of five microbial species to alkaline stress. J Endod 2008;34:579- 582. 